\section{Nonalcoholic fatty liver disease is associated with coronary artery disease and subclinical atherosclerosis}

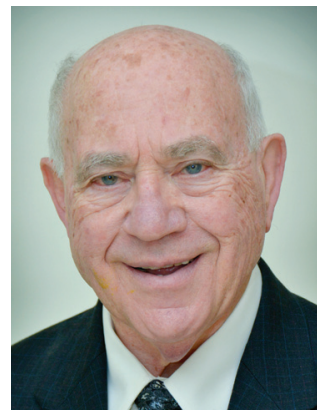

"Nonalcoholic fatty liver disease is fatty infiltration of the liver in absence of significant alcohol intake, steatogenic medication or hereditary disorders and is a common cause of chronic liver disease."

Wilbert S Aronow*

First draft submitted: 9 April 2016; Accepted for publication: 25 April 2016;

Published online: 13 June 2016

Nonalcoholic fatty liver disease (NAFLD) is fatty infiltration of the liver in absence of significant alcohol intake, steatogenic medication or hereditary disorders and is a common cause of chronic liver disease. NAFLD is a liver manifestation of the metabolic syndrome [1]. Insulin resistance and increased oxidative stress predispose to NAFLD, and risk factors for NAFLD include metabolic syndrome, diabetes, obesity, lack of physical activity, smoking and a high-fat diet. Cardiovascular disease is a major cause of mortality in patients with NAFLD.

Among 612 patients undergoing coronary angiography, NALFD diagnosed by ultrasonography was present in 58\% of patients [2]. Coronary artery disease (CAD) was diagnosed by coronary angiography in $84.6 \%$ of patients with NAFLD and in $64.1 \%$ of patients without NALFD $(\mathrm{p}<0.001)$. Patients with NAFLD had a 2.32-times higher prevalence of CAD than those without NAFLD independent of demographic and metabolic factors [2]. At 5-year follow-up of 2103 patients with
Type 2 diabetes, those with NAFLD developed an 84\% higher incidence of cardiovascular disease (CAD, ischemic stroke or death from cardiovascular causes) than those without NAFLTD ( $<$ 0.001) [3]. The increased development of cardiovascular disease in patients with NAFLTD was independent of cardiovascular risk factors [3].

A prospective observational study included 1637 healthy Japanese men and women who were followed for 5 years [4]. Among 1221 persons available for analysis of outcomes, the incidence of atherosclerotic cardiovascular disease (CAD, ischemic stroke and cerebral hemorrhage) was higher in persons with NAFLD at baseline than those without NAFLD. Multivariate analyses demonstrated that NAFLD predicted cardiovascular disease independent of cardiovascular risk factors (odds ratio: 4.12; $\mathrm{p}=0.004)$ [4]. Both NAFLD and the metabolic syndrome predicted cardiovascular disease, but only NAFLD showed a significant correlation with cardiovascular disease by multivariate analysis [4].

*Cardiology Division, Department of Medicine, Westchester Medical Center/New York Medical College, Valhalla, NY, USA; Tel.: +1 914493 5311; Fax: +1 914235 6274; wsaronow@aol.com

\section{KEYWORDS}

- atherosclerotic cardiovascular disease $\bullet$ brachial-ankle pulse wave velocity $\bullet$ cardioankle vascular index • carotid intimamedia thickness $\bullet$ coronary artery calcification - coronary artery disease - nonalcoholic fatty liver disease

"Many studies have shown an association between nonalcoholic fatty liver disease and subclinical atherosclerosis. Most of these studies prove that this association is independent of metabolic syndrome and cardiovascular risk factors." 


\section{"Even though strong epidemiological evidence is available linking nonalcoholic fatty liver disease with atherosclerotic vascular disease and subclinical atherosclerosis, our knowledge of the biochemical pathways contributing to this association is incomplete."}

Pisto et al. followed 988 persons from 1991-2009 [5]. During follow-up, $13.5 \%$ of patients with a nonfatty liver, $24.2 \%$ of patients with moderate liver fat content and $29.2 \%$ of patients with severe fatty liver content developed a cardiovascular event $(p<0.001)$. Severe hepatic fat content was predictive of future cardiovascular events when adjusted for age, gender and study group (hazard ratio: 1.92; $\mathrm{p}<0.01$ ). The risk for future cardiovascular events remained significant (hazard ratio: 1.74; $\mathrm{p}<0.01$ ) after additional adjustment for smoking, use of alcohol, serum LDL cholesterol, BMI and systolic blood pressure [5].

The association of NAFLD with CAD was investigated in 273 Type 2 diabetic patients undergoing coronary computed tomography for chest pain [6]. After adjustment for age, gender, obesity, hypertension, smoking and serum LDL cholesterol, NAFLD was associated with significant CAD (odds ratio: $2.128 ; p=0.04$ ) [6].

A prospective study of 80 patients with the metabolic syndrome undergoing coronary angiography investigated the association between NAFLD diagnosed by abdominal ultrasonography and the severity of CAD [7]. Coronary angiography showed involvement of more vessels $(2.5 \pm 0.9$ vs $1.0 \pm 1.0 ; \mathrm{p}<0.001)$ and more severe CAD diagnosed by Gensini scores $(\mathrm{p}<0.001)$ among patients who had NAFLD. Multivariate regression analysis showed that NAFLD was the only independent factor affecting the CAD severity score [7].

Another study investigated a cross-sectional analysis of 355 patients undergoing coronary angiography and abominal ultrasonography for NAFLD [8]. Multivariate analysis showed that NAFLD was independently associated with the prevalence of CAD (odds ratio: 2.58; $\mathrm{p}<0.01$ ) and also independently with severity of CAD measured by the Gensini score (odds ratio: 2.02; $\mathrm{p}<0.05)[8]$.

Sun et al. performed abdominal computed tomography to detect NAFLD in 542 patients before undergoing coronary angiography for suspected CAD [9]. Logistic regression analysis showed that NAFLD independently increased the risk of CAD seen on coronary angiography (odds ratio: 7.585; $\mathrm{p}<0.001$ ). NAFLD was significantly more prevalent in patients as the severity of CAD increased [9]. These investigators concluded that patients with NAFLD should be closely followed for not only presence of CAD, but also for its severity [9].
Coronary collateral development has been shown to improve survival in patients with CAD. The metabolic syndrome and associated insulin resistance has been shown to be associated with poor coronary collateral development. A study investigated 151 nondiabetic patients with stable angina pectoris who had more than $95 \%$ stenosis in at least one major coronary artery [10]. Abdominal ultrasonography performed after coronary angiography demonstrated that NAFLD was present in $65 \%$ of study patients and was more prevalent in patients with poor collateral development $(82.9 \%$ in NAFLD patients vs $49.4 \%$ in non-NAFLD patients, $\mathrm{p}<0.001)$. The mean Rentrop collateral score was significantly lower in patients with NAFLD than in patients without NAFLD ( $\mathrm{p}<0.001)$. NAFLD was significantly related to poor circulation (odds ratio: 6.20; 95\% CI: 2.61-14.75) after logistic regression analysis was performed using factors associated with poor collateral development [10].

Many studies have shown an association between NAFLD and subclinical atherosclerosis. Most of these studies prove that this association is independent of metabolic syndrome and cardiovascular risk factors.

Coronary artery calcification (CAC) is a surrogate marker for atherosclerotic burden and an independent marker of CAD risk. Data were analyzed from 10,153 individuals who received an ultrasound of the abdomen for assessment of fatty liver and a cardiac computerized tomography computed CAC score [11]. Fatty liver was associated with a CAC score $>0$ independent of metabolic syndrome, coronary risk factors and previous cardiovascular disease (odds ratio: 1.21; $\mathrm{p}=0.04)$ [11]. The CARDIA study included 2424 patients, mean age 50 years, which used computed tomography to quantify liver fat, CAC and abdominal aortic calcification [12]. Patients with NAFLD had increased prevalence of CAC (37.9 vs $26.0 \%$; $p<0.001)$ and abdominal aortic calcification ( 65.1 vs $49.9 \%$; $\mathrm{p}<0.001)$. This association of NAFLD with CAC and abdominal aortic calcification persisted after adjustment for demographics and health behaviors [12].

A study involved a cross-sectional analysis of 3796 persons in the MESA study who had computed tomography imaging [13]. This study showed that NAFLD is associated with increased inflammation measured by high sensitivity C-reactive protein $\geq 2 \mathrm{mg} / \mathrm{l}$ (odds ratio: 1.47; 95\% CI: $1.20-1.79)$ and CAC greater than 0 
(odds ratio: 1.37; 95\% CI: 1.11-1.68) independent of coronary risk factors, obesity, and metabolic syndrome [13]. There was a graded association among NAFLD, obesity and metabolic syndrome with inflammation and CAC [13].

Measurement of carotid intima-media thickness (CIMT) using ultrasound is a marker of subclinical atherosclerosis. CIMT is a strong predictor of future vascular events and better predicts the risk of stroke compared with myocardial infarction. A cross-sectional study showed that subjects with NAFLD had higher average and maximum CIMT than controls [14]. NAFLD was an independent predictor of having high average CIMT (odds ratio: 4.8; 95\% CI: 1.8-12.8) and high maximum CIMT (odds ratio: 5.4 ; $95 \%$ CI: $2.0-14.4$ ) after adjusting for obesity, metabolic syndrome, insulin resistance and lipid parameters [14].

A large cross-sectional study of 8632 persons revealed a significantly higher CIMT $(\mathrm{p}<0.0001)$ and brachial-ankle pulse wave velocity $(b$ PWV) $(p<0.0001)$ in persons with NAFLD compared with those without NAFLD [15]. Logistic regression analysis showed that NAFLD conferred a $35 \%$ increased odds ratio of elevated CIMT and a 30\% increased odds ratio of $b_{2}$ PWV independent of coronary risk factors and the metabolic syndrome [15].

A meta-analysis of ten studies including 1947 patients showed that pathological CIMT was present in $35.1 \%$ of NAFLD patients compared with $21.8 \%$ of patients without NAFLD $(\mathrm{p}<0.0001)$ [16]. A meta-analysis of four studies including 1198 patients showed that CAD diagnosed by coronary angiography was present in $80.4 \%$ of patients with NAFLD versus $60.7 \%$ of patients without NAFLD $(\mathrm{p}<0.001)$ [16].

Another measure of arterial stiffness is b PWV. A prospective study of 728 men and 497 women free of hypertension and diabetes investigated progression of arterial stiffening by measuring $b_{a}$ PWV [17]. At 5-year follow-up, multiple regression analysis revealed that NAFLD is an independent and significant predictor for progression of $b_{a}$ PWV $(p<0.001)$ in men and in women after adjustment for cardiovascular risk factors [17].

The cardioankle vascular index (CAVI) indicates stiffness of the entire arterial segment from aorta to the ankle. A study of 443 patients undergoing CAVI, carotid ultrasonography and coronary angiography showed by multiple logistic analyses that high CAVI indicates progression of CAD and carotid arteriosclerosis [18]. CAVI also predicts stroke [18,19]. In a cross-sectional analysis of 2954 subjects, NAFLD was associated with a $42 \%$ increase in risk of arterial stiffness [20]. The risk of arterial stiffness increased with severity of NAFLD. The association was statistically significant after adjustment for other risk factors including BMI, waist circumference, smoking status, diabetes and hypertension [20].

Even though strong epidemiological evidence is available linking NAFLD with atherosclerotic vascular disease and subclinical atherosclerosis, our knowledge of the biochemical pathways contributing to this association is incomplete. More studies are needed to understand the underlying biochemical pathways and markers responsible for the above clinical findings, which could lead to development of needed therapeutic interventions.

\section{Financial \& competing interests disclosure}

The author has no relevant affliations or financial involvement with any organization or entity with a financial interest in or financial conflict with the subject matter or materials discussed in the manuscript. This includes employment, consultancies, honoraria, stock ownership or options, expert testimony, grants or patents received or pending, or royalties.

No writing assistance was utilized in the production of this manuscript.

\section{References}

1 Milić S, Lulić D, Štimac D. Non-alcoholic fatty liver disease and obesity: biochemical, metabolic and clinical presentations. World J. Gastroenterol. 20(28), 9330-9337 (2014).

2 Wong VW, Wong GL, Yip GW et al. Coronary artery disease and cardiovascular outcomes in patients with non-alcoholic fatty liver disease. Gut 60(12), 1721-1727 (2011)
3 Targher G, Bertolini L, Rodella S et al. Nonalcoholic liver disease and risk of future cardiovascular events among Type 2 diabetic patients. Diabetes 54(12), 3541-3546 (2005).

4 Hamaguchi M, Kojima T, Takeda N et al. Nonalcoholic fatty liver disease is a novel predictor of cardiovascular disease. World J. Gastroenterol. 13(10), 1579-1584 (2007).

5 Pisto P, Santaniemi M, Bloigu R et al. Fatty liver predicts the risk for cardiovascular events in middle-aged population: a population- based cohort study. BMJ Open 4(3), e004973 (2014).

6 Idilman IS, Akata D, Hazirolan T et al. Nonalcoholic fatty liver disease is associated with significant coronary artery disease in Type 2 diabetic patients: a computed tomography angiography study 2 . J. Diabetes 7(2), 279-286 (2015).

7 Alper AT, Hasdemir H, Sahin S et al. The relationship between nonalcoholic fatty liver disease and the severity of coronary artery 


\section{EDITORIAL Aronow}

disease in patients with metabolic syndrome. Turk. Kardiyol. Dern. Ars. 36(6), 376-381 (2008).

8 Açikel M, Sunay S, Koplay M et al. Evaluation of ultrasonographic fatty liver and severity of coronary atherosclerosis, and obesity in patients undergoing coronary angiography. Anadolu. Kardiyol. Derg. 9(4), 273-279 (2009).

9 Sun L, Lü SZ. Association between non-alcoholic fatty liver disease and coronary artery disease severity. Chin. Med. J. (Engl.). 124(6), 867-872 (2011).

10 Arslan U, Kocaoğlu I, Balcı M et al. The association between impaired collateral circulation and non-alcoholic fatty liver in patients with severe coronary artery disease. J. Cardiol. 60 (3), 210-214 (2012).

11 Sung KC, Wild SH, Kwag HJ, Byrne CD. Fatty liver, insulin resistance, and features of metabolic syndrome: relationships with coronary artery calcium in 10,153 people. Diabetes Care 35(11), 2359-2364 (2012).
12 VanWagner LB, Ning H, Lewis CE et al. Associations between nonalcoholic fatty liver disease and subclinical atherosclerosis in middle-aged adults: the Coronary Artery Risk Development in Young Adults Study. Atherosclerosis 235(2), 599-605 (2014).

13 Al Rifai M, Silverman MG, Nasir K et al. The association of nonalcoholic fatty liver disease, obesity, and metabolic syndrome, with systemic inflammation and subclinical atherosclerosis: the Multi-Ethnic Study of Atherosclerosis (MESA). Atherosclerosis 239(2), 629-633 (2015).

14 Thakur ML, Sharma S, Kumar A et al. Nonalcoholicfatty liver disease is associated with subclinical atherosclerosis independent of obesity and metabolic syndrome in Asian Indians. Atherosclerosis 223(2), 507-511 (2012).

15 Huang Y, Bi Y, Xu M et al. Nonalcoholic fatty liver disease is associated with atherosclerosis in middle-aged and elderly Chinese. Arterioscler. Thromb. Vasc. Biol. 32(9), 2321-2326 (2012).
16 Ampuero J, Gallego-Durán R, RomeroGómez M. Association of NAFLD with subclinical atherosclerosis and coronary-artery disease: meta-analysis. Rev. Esp. Enferm. Dig. 107(1), 10-16 (2015).

17 Li N, Zhang GW, Zhang JR et al. Nonalcoholic fatty liver disease is associated with progression of arterial stiffness. Nutr. Metab. Cardiovasc. Dis. 25(2), 218-223 (2015).

18 Izuhara M, Shioji K, Kadota $S$ et al. Relationship of cardio-ankle vascular index (CAVI) to carotid and coronary arteriosclerosis. Circ. J. 72(11), 1762-1767 (2008).

19 Suzuki J, Sakakibara R, Tomaru T et al. Stroke and cardio-ankle vascular stiffness index. J. Stroke Cerebrovasc. Dis. 22(2), 171-175 (2013).

20 Chung GE, Choi SY, Kim D et al. Nonalcoholic fatty liver disease as a risk factor of arterial stiffness measured by the cardioankle vascular index. Medicine (Baltimore) 94(12), e654 (2015). 